\title{
Investigación textil en España: un caso de aplicación de indicadores bibliométricos a nivel micro
}

\author{
Enric Carrera-Gallissà๋, Xavier Capdevila*
}

Resumen: El estudio analiza, por primera vez, el indice $b$ de los investigadores textiles de un país (España) y aporta información, también por primera vez, del rango de valores de este índice en el área de conocimiento textil. Se han estudiado también 10 indicadores de la producción científica de los investigadores textiles en España entre 1955 y 2009. La población objeto de este estudio está formada por 73 personas pertenecientes a 6 instituciones distintas. La población de investigadores textiles es un colectivo heterogéneo ya que éstos pertenecen a áreas de conocimientos distintos. El índice $b$ estudiado presenta una correlación positiva, entre intensa y notable, con el número de publicaciones, publicaciones por año y número de citas recibidas, citas por año, número de citas recibidas por la publicación más citada. La correlación es notable pero de signo negativo con el porcentaje de publicaciones de la obra del autor que no han sido citadas nunca. El Consejo Superior de Investigaciones Científicas (CSIC) presenta niveles superiores, respecto a las otras instituciones, en los siguientes indicadores: índice $h$, número de publicaciones, citas, citas por año y máximo de citas de la publicación más citada. El Instituto Tecnológico Textil de Alcoy (AITEX) tiene los mayores niveles en los siguientes indicadores: indice $b$ por años de actividad y porcentaje de publicaciones no citadas. Finalmente, se relaciona el índice $b$ con tres factores (latentes) que explican los otros indicadores y proporcionan tres dimensiones de aquel.

Palabras clave: Indice $h$, Investigadores textiles, España, Indicadores bibliométricos.

\section{Textile-related research in Spain: a case study applying micro bibliometric indicators}

Abstract: The study examines the b-index of textile researchers in Spain and, for the first time, provides information, on the range of values of this index in the area of textilerelated knowledge. This paper reports on a study of 10 scientific production indicators for Spanish textile researchers over the period 1955-2009. The population studied consisted of 73 researchers affiliated with 6 different institutions. Textile investigators represent a beterogeneous community, since they come from different areas of knowledge. The $b$-index studied presents a positive correlation, ranging between intense and notable, with the number of publications, publications by year, number of citations, citations per year, number of citations received by the most cited publication. The correlation is significant but negative concerning the percentage of authors' non-cited publications.

* Departamento de Ingeniería Textil y Papelera, Universitat Politècnica de Catalunya, EET, Terrassa, España. Correo-e: carrera@catunesco.upc.edu; xcapdevila@etp.upc.edu. 
The Consejo Superior de Investigaciones Cientificas (CSIC) has higher levels than the other institutions for the following indicators: $b$-index, number of publications, citations, citation per year, maximum number of citations. The Instituto Tecnológico Textil de Alcoy (AITEX) has the bighest levels for the following indicators: $b$-index per year of activity and percentage of non-cited publications. Finally, a relation is shown between the $b$-index and three (latent) factors that explain the other indicators and provide three dimensions of the $b$-index.

Keywords: $h$ Index, Spain, Textile researchers, Bibliometrics indicators

\section{Introducción}

El uso de la literatura científica como una medida de la actividad investigadora tiene una gran tradición en el ámbito científico (Pritcchard, 1969). El tratamiento de esta información por medios cuantitativos de recuento y análisis tiene un gran interés no sólo para analizar el volumen de publicaciones, la productividad de los autores, revistas o materias sino también para el conocimiento de los procesos y la naturaleza de las ciencias, siendo a partir de 1960 cuando empieza a acuñarse el término de Cienciometría.

Si bien existen indicadores bibliométricos de circulación que miden la presencia de documentos en las bibliotecas y bases de datos bibliográficas, indicadores que miden la dispersión de la información científica, indicadores sobre la vida media de la literatura circulante, indicadores sobre la dimensión cuantitativa de la producción científica, los que presentan mayor interés son los relacionados con la dimensión cualitativa de la producción científica. De éstos últimos la evaluación de la calidad de la producción científica se fundamenta en la cita como elemento clave y núcleo central de su corpus doctrinal. Uno de los más conocidos es el Factor de Impacto (Garfield, 1955) de las revistas donde se publica la información. A pesar de que se trata de un indicador interesante presenta bastantes limitaciones y contradicciones lo cual ha dado pie a una abundante literatura (Buela-Casal, 2001) (Buela-Casal, 2002) (Buela-Casal, 2003),(Buela-Casal y otros, 2006) (Garfield, 1984) (Garfield, 1996) (Nieminen y otros, 2006) (Seglen, 1998) (Seglen, 1997) (Simkim y otros, 2003).

La aparición, hace tan sólo seis años, del indice $h$, un nuevo e ingenioso método de medición de la calidad profesional de físicos y otros científicos propuesto por el profesor J. E. Hirsch de la Universidad de California (Hirsch, 2005), ha provocado una auténtica revolución entre los investigadores de todas las ciencias.

El indice $h$, que fue diseñado inicialmente para medir eficazmente la calidad de un investigador, es el balance entre el número de publicaciones y las citas de éstas. Así, por ejemplo, un científico tiene indice $b$ equivalente a 8 si ha publicado 8 trabajos con al menos 8 citas cada uno, pero no tiene 9 publicaciones que hayan sido citadas 9 veces cada una de ellas.

Diversos autores (Iglesias y otros, 2006) (Costas y Bordons, 2007), comenzando por el propio Hirsch (2005) han señalado que el indice $h$, tomado en valor 
absoluto, a puede ser usado para comparar investigadores de ciencias diferentes debido a los distintos hábitos de publicación y citación según el campo.

Según Imperial y Rodríguez-Navarro (2009) los trabajos en las ciencias aplicadas reciben menos citas que los trabajos en las ciencias más básicas y, en consecuencia, los científicos en las disciplinas aplicadas tienen valores $b$ menores que los de las disciplinas básicas. Esto se debe a la dependencia del indice $b$ del tamaño de la población de investigadores, ya que cuanto mayor es la población más posibilidades de que el índice $b$ también sea mayor al existir mayor posibilidad de citas

La gran ventaja de éste indicador respecto a los otros existentes hasta la fecha es que combina una medida de cantidad y de impacto en un solo indicador, superando los recuentos brutos de documentos y citas, a lo que hay que añadir su sencillez de cálculo (Costas y Bordons, 2007).

De entre todas las limitaciones del indice $h$, hay dos que sin lugar a dudas son cruciales.

a) la dependencia entre el indice de un investigador y su número total de publicaciones, de forma que el indice $b$ nunca podrá ser superior al número total de documentos. De este modo, una persona con 20 publicaciones nunca podrá tener un indice $b$ superior a 20, independientemente del número de citas que reciban sus artículos.

b) los trabajos muy citados contribuyen al indice $b$ de un determinado autor, pero el número de veces que son citados no influye sobre su valor.

En cualquier caso, algunas agencias nacionales de evaluación de la actividad científica, como Brasil, ya lo han adoptado como uno de sus criterios para la asignación de recursos financieros (Salgado y Páez, 2007).

\section{Objetivos}

En todo el mundo los investigadores textiles configuran un colectivo profesional muy heterogéneo debido al amplísimo espectro de ámbitos de estudio que abarca esta área de conocimiento. Esta singularidad dificulta la evaluación de la actividad investigadora de este colectivo en la medida que la mayoría de los indicadores bibliométricos clásicos no pueden ser utilizados para comparar ámbitos de estudio distintos, Seglen (1997 y 1998), Hirsch (2005), Iglesias y Pecharromán (2006), Imperial y Rodríguez-Navarro (2009), etc. A pesar de ello algunas Universidades españolas, como la Universitat Politècnica de Catalunya, han empezado a utilizar a nivel interno y de forma experimental el indice $b$ como un descriptor más de la carrera profesional de su personal docente e investigador. Ante el riesgo que representa la utilización única de ése indicador en colectivos profesionales tan heterogéneos como el textil y ante la falta de referencias bibliográficas a nivel mundial (no se han encontrado ningún artículo en el Web os Science que hable 
del indice $b$ en el área textil), sobre el rango de valores que presentan los investigadores de ésta área, se ha realizado un primer estudio del índice $b$ de los investigadores textiles españoles con el objetivo de realizar una primera aportación que contribuya a conocer la variabilidad de éste indicador en ésta área de conocimiento. A pesar de que se trata de un estudio a nivel micro (sólo se estudia la realidad española) la información que se utiliza se ha obtenido de la población objeto de estudio y no de una muestra como la mayoría de estudios similares. Por otra parte hay que recordar que España es un país con una industria textil de larga tradición y con una formación universitaria y actividad investigadora notable.

\section{Metodología}

El horizonte temporal del estudio se extiende entre 1955 y 2009, y la población objeto de medición es:

a) Personal docente e investigador de las universidades españolas que imparten docencia de ingeniería textil: Universitat Politècnica de Catalunya (Departamento de Ingeniería Textil y Papelera, Departamento de Ingeniería Química, Instituto de Investigación Textil y de Cooperación Industrial de Terrassa y Departamento de Estadística e Investigación operativa) -UPC- Universidad Politécnica de Valencia (Campus Alcoy) -UPV- y Universidad de Salamanca (Campus Béjar) -US.

b) Personal investigador de las siguientes instituciones: Consejo Superior de Investigaciones Científicas de Barcelona -CSIC-, Instituto Tecnológico Textil de Alcoy -AITEX- Asociación de Investigación Textil Algodonera de Barcelona (hoy extinta) -AITA.

Los indicadores estudiados son: indice $b(\mathbf{h})$, indice $b$ por años de actividad (m), número de publicaciones (nP), número de publicaciones por año de acti$\operatorname{vidad}(\mathbf{P a})$, número de citas recibidas (c), número de citas por publicación (cP), años transcurridos entre la primera y la última publicación incluida en ISI (a), número de citas por año de actividad (c/a), número de citas recibidas por la publicación más citada (McP), porcentaje de publicaciones de la obra del autor que no han sido citadas nunca (\% NC).

Los indicadores se obtuvieron a través del análisis de la documentación existente en el Web of Science de ISI. La búsqueda se realizó incluyendo las siguientes bases de datos:

- Science Citation Index Expanded (SCI-EXPANDED). Desde 1900 hasta 2009.

- Social Sciences Citation Index (SSCI). Desde 1956 hasta 2009.

- Arts \& Humanities Citation Index (A\&HCI). Desde 1975 hasta 2009.

- Conference Proceedings Citation Index- Science (CPCI-S). Desde 1990 hasta 2009. 
- Conference Proceedings Citation Index- Social Science \& Humanities (CPCISSH). Desde 1990 hasta 2009.

- Index Chemicus (IC). Desde 1993 hasta 2009.

- Current Chemical Reactions (CCR-EXPANDED). Desde 1986 hasta 2009.

El cálculo del índice $b$ de cada autor se obtuvo a partir del nombre y apellidos del autor, realizando una posterior depuración por país (España) e institución a la que pertenece o ha pertenecido cada autor.

Las consultas de toda esta información se realizaron entre los días 12 y 18 de noviembre de 2009.

Un dilema importante que se ha encontrado durante la realización de este estudio ha sido definir con claridad y precisión el concepto de investigador textil y sus límites. Se trata, éste, de un ámbito extremadamente amplio y heterogéneo que incluye un amplio espectro de áreas de conocimiento, tanto procesos agrícolas o ganaderos como son la producción de fibras naturales, largos procesos de química industrial como la obtención de fibras químicas y las propiedades de sus polímeros componentes, pasando por los procesos propiamente textiles de hilatura, tisaje, tricotado, descrudado, blanqueo, tintura, estampado, ennoblecimiento, detergencia y confección o bien de aspectos ambientales como el tratamiento y depuración de aguas, gases o residuos textiles. Se han incluido también los temas relacionados con el desarrollo de nuevos productos, técnicas analíticas, métodos de ensayo o bien el control de calidad ya sea de los productos textiles acabados, productos intermedios del proceso textil o bien los fundamentos físicos y químicos del amplísimo espectro de productos auxiliares utilizados en el proceso textil dado que también son objeto habitual de la actividad de los investigadores textiles. A todo ello habría que añadir los ámbitos relacionados con la gestión, marketing o comercio de productos textiles y auxiliares del proceso.

Dado que muchos de los investigadores estudiados trabajan en el terreno fronterizo de los que es lo propiamente textil o bien en la investigación básica que fundamenta las aplicaciones y desarrollos textiles se ha considerado a efectos de este estudio como investigador textil aquella persona que al menos el $25 \%$ del total de sus publicaciones son claramente textiles o bien se han publicado en las revistas textiles más importantes de ISI.

\section{Resultados y discusión}

Un total de 73 investigadores textiles en España reúnen los requisitos descritos anteriormente, 31 de los cuales pertenecen a la Universidad Politécnica de Cataluña (UPC), 12 a la Universidad Politécnica de Valencia (UPV), 2 a la Universidad de Salamanca (US), 19 al centro de investigación del Consejo Superior de Investigaciones Científicas (CSIC) en Barcelona (antiguamente denominado Instituto de Tecnología Química y Textil), 7 al Instituto Tecnológico Textil AITEX, y 2 a la Asociación de Investigación Textil Algodonera (AITA). 
Si bien el número de profesores/as de ingeniería textil en España es algo mayor que las cifras indicadas anteriormente, hay que tener presente que no todos ellos han publicado en revistas incluidas en el Journal of Citation Reports. En cualquier caso, los 73 investigadores estudiados constituyen la población de este colectivo y no una muestra.

Durante el período estudiado la producción de los investigadores textiles españoles ascendió a 1.833 documentos. La productividad osciló entre 0 y 181 trabajos, variando su impacto entre 0 y 15,80 citas por documento, y el índice $b$ de los científicos entre 0 y 21.

La tabla I presenta el análisis de los diversos indicadores bibliométricos de la producción científica de los investigadores textiles.

\section{TABLA I}

Indicadores bibliométricos de los investigadores textiles españoles

\begin{tabular}{l|c|c|c|c|c|c|c|c|c|c}
\hline & $\mathbf{h}$ & $\mathbf{m}$ & $\mathbf{n P}$ & $\mathbf{P a}$ & $\mathbf{C}$ & $\mathbf{c P}$ & $\mathbf{a}$ & $\mathbf{c} / \mathbf{a}$ & $\mathbf{M c P}$ & $\mathbf{\%} \mathbf{~ N C}$ \\
\hline $\mathrm{N} .{ }^{\circ}$ & 73 & 73 & 73 & 73 & 73 & 73 & 73 & 73 & 73 & 73 \\
\hline $\mathrm{Me}$ & 5,38 & 0,44 & 25,11 & 1,45 & 171,63 & 5,42 & 15,58 & 9,10 & 23,30 & 30,02 \\
\hline $\mathrm{S}$ & 4,68 & 0,31 & 36,54 & 1,24 & 328,07 & 3,99 & 11,54 & 11,66 & 22,40 & 26,79 \\
\hline $\mathrm{CV}$ & 87,05 & 70,75 & 145,55 & 85,77 & 191,15 & 73,73 & 74,09 & 128,19 & 96,15 & 89,26 \\
\hline Mín. & 0 & 0 & 1 & 0,15 & 0 & 0 & 1 & 0 & 0 & 0 \\
\hline Máx. & 21 & 1 & 181 & 6,42 & 1926 & 17,22 & 43 & 56,64 & 108 & 100 \\
\hline $\mathrm{R}$ & 21 & 1 & 180 & 6,27 & 1926 & 17,22 & 42 & 56,64 & 108 & 100 \\
\hline $\mathrm{A}$ & 1,34 & 0,58 & 2,61 & 2,02 & 3,63 & 0,88 & 0,55 & 2,14 & 1,61 & 1,30 \\
\hline $\mathrm{K}$ & 1,51 & $-0,84$ & 7,09 & 4,37 & 15,20 & 0,54 & $-0,69$ & 4,96 & 2,98 & 1,23 \\
\hline
\end{tabular}

N. ${ }^{\circ}$ : número de investigadores, Me: promedio, S: desviación estándar, CV: coeficiente de variación (\%), Mín.: valor mínimo, Máx.: valor máximo, R: rango, A: coeficiente de asimetría, K: coeficiente de Curtosis.

Los resultados de la tabla I — calculados con el programa Minitab- muestran, en primer lugar, un coeficiente de variación muy elevado en todos los indicadores (expresa la desviación estándar como un porcentaje del valor absoluto de la media, y es superior a 50 en todos los indicadores). Se observa asimismo distribuciones con asimetría positiva (todas los coeficientes A distintos de cero, aunque $\mathrm{m}$ y a son poco asimétricas), distribuciones leptocúrticas en los indicadores $\mathrm{h}$, $\mathrm{nP}, \mathrm{Pa}, \mathrm{c}, \mathrm{c} / \mathrm{a}, \mathrm{McP}$ y \% NC (aunque el grado de "apuntamiento" es muy acentuado en las distribuciones de $\mathrm{nP}$ y c) y distribuciones casi mesocúrtica en $\mathrm{m}, \mathrm{cP}$ y a. Gráficamente, las distribuciones se muestran mediante diagramas de cajas (el extremo inferior de la caja es el primer cuartil, el superior el tercero y la línea central la mediana o segundo cuartil) en las figuras 1 a 10.

En cualquier caso el indice $b$ medio de los investigadores textiles españoles es de 5,38, siendo su valor máximo 21 y el mínimo 0. 


\section{FIGURA 1}

Diagrama de caja del índice $b$

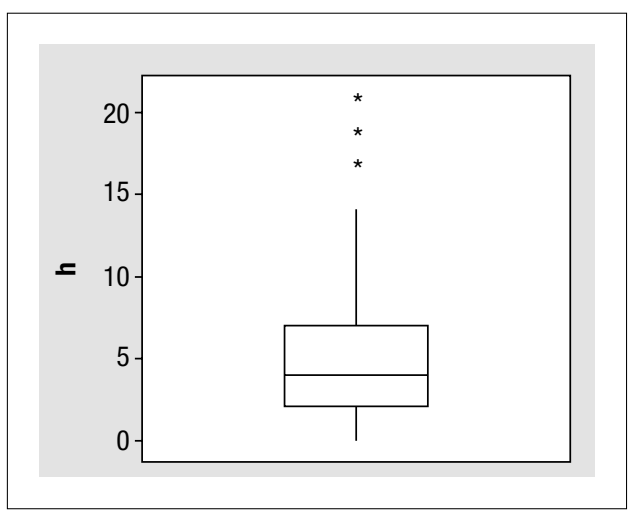

FIGURA 3

Diagrama de caja del indicador $n P$

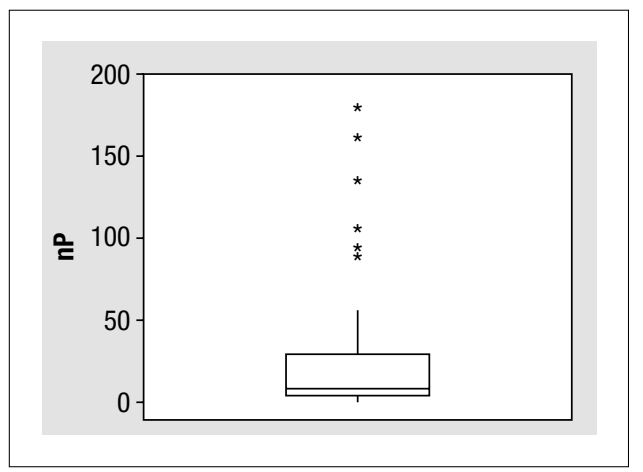

FIGURA 5

Diagrama de caja del indicador c

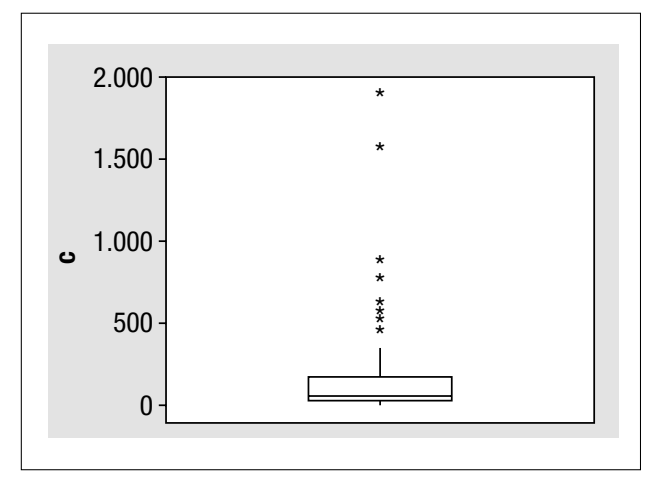

FIGURA 2

Diagrama de caja del indicador $m$

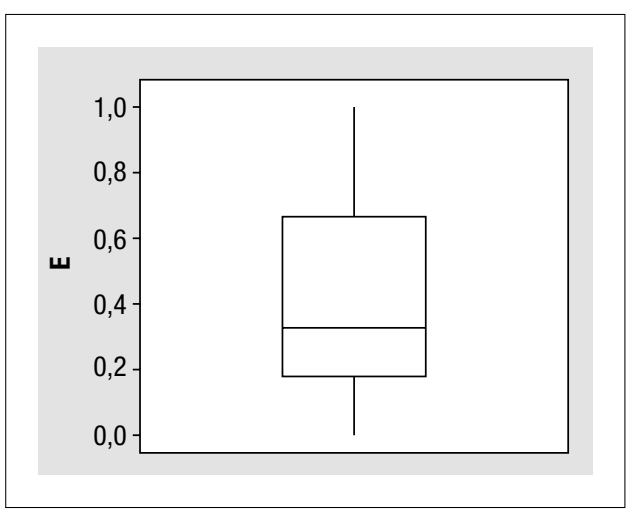

FIGURA 4

Diagrama de caja del indicador $\mathrm{Pa}$

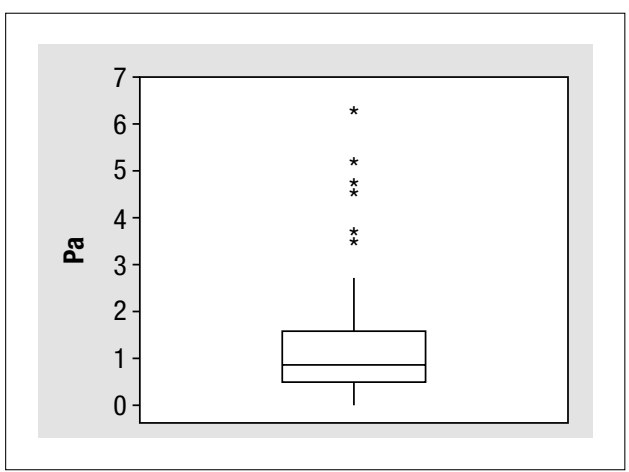

FIGURA 6

Diagrama de caja del indicador $c P$

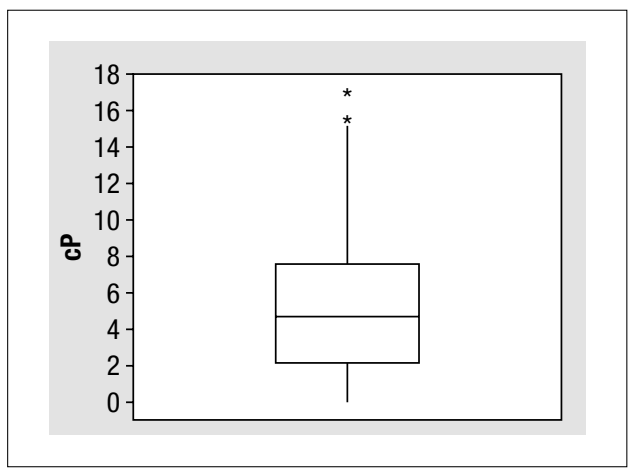


FIGURA 7

Diagrama de caja del indicador a

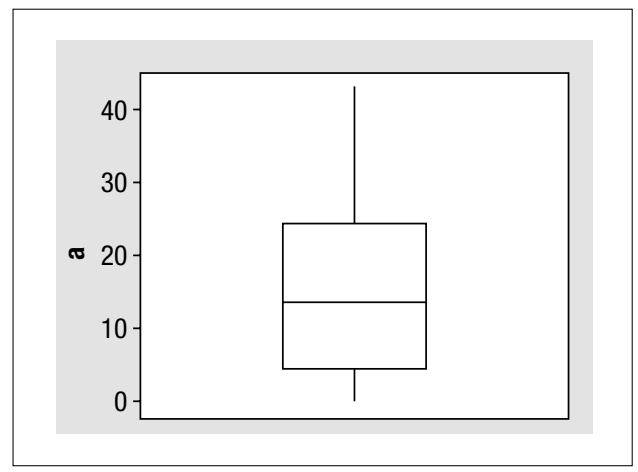

FIGURA 9

Diagrama de caja del indicador $\mathrm{McP}$

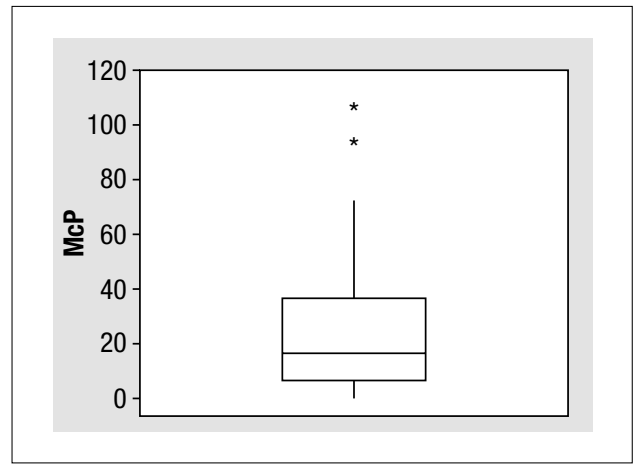

FIGURA 8

Diagrama de caja del indicador $c / a$

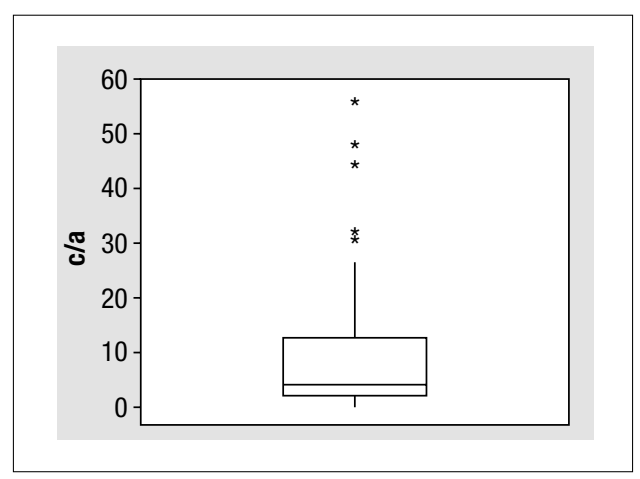

FIGURA 10

Diagrama de caja del indicador $\%$ NC

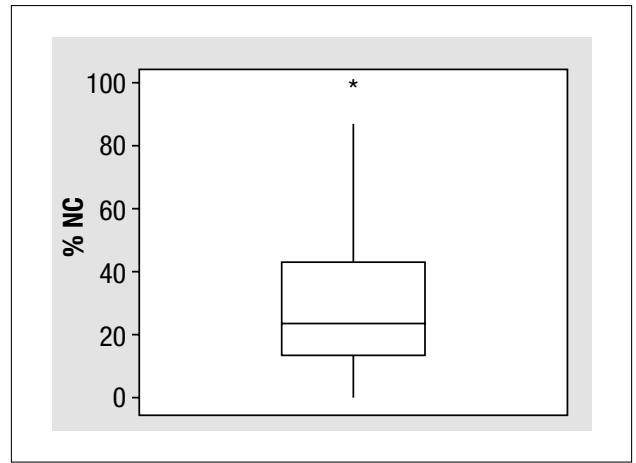

Aunque los valores obtenidos en este estudio son relevantes, la ausencia de estudios similares en otros países y en el ámbito textil dificulta notablemente la valoración de los resultados obtenidos para España.

Los resultados del presente estudio son concordantes con la elevada hetereogenidad del colectivo estudiado, tanto por los años de trayectoria de sus miembros como por la tipología del investigador (si la investigación es o no, compartida con la docencia u otras actividades), como por el área de conocimiento o ámbito de investigación dentro del sector textil en el que trabajan principalmente.

El indice $m$ propuesto por Hirsch (2005) divide $b$ entre la edad científica de los investigadores - en nuestro caso los años transcurridos entre la primera y la última publicación registrada en ISI - de tal forma que $m$ puede considerarse, en el fondo, como la velocidad a la cual aumenta el indice $b$ de un investigador (Kelly y Jennions, 2006). También conviene señalar que el indicador «a» (años), 
hace referencia al período transcurrido entre la primera y la última de las publicaciones incluidas en ISI.

El porcentaje de publicaciones de la obra de cada investigador que no han sido citados nunca (\% NC) visualiza en cierta medida el impacto, utilidad y contribución de la investigación realizada en el avance de la ciencia, así como del reconocimiento de la obra de un autor entre los compañeros de profesión. En este estudio se puede afirmar que más de una cuarta parte de la investigación textil española $(30,02 \%)$, no ha sido citada nunca. Aunque existen pocos estudios al respecto, todo parece indicar que estamos ante un valor habitual. Seglen (1992), afirma que la mayoría de los trabajos no suelen ser citados nunca o casi nunca, y que el 15\% de los artículos publicados en una revista acapara el 50\% de todas las citas. El propio Institute of Scientific Information indicaba en un informe de 1999 que el porcentaje de artículos no citados oscilaba entre el 44,5\% de Ciencias de la Computación, el 25\% en Psicología y Psiquiatría y el 13\% de Inmunología (ISI, 1999).

Es difícil realizar una evaluación del resto de indicadores debido a la ausencia de referencias publicadas para ésta área de conocimiento. Sin embargo, del indicador Pa podemos deducir que la producción científica de esta área en España es más bien baja en relación con otras áreas (véase clasificación de los investigadores españoles según el índice $b$ realizada por Luis Pérez en la web: http://indice-h.webcindario.com/) dado que el valor medio de Pa es de 1,45 (suficiente para obtener un sexenio de investigación), con un valor máximo de 6,42. Esta misma tendencia se observa al analizar el número de citas recibidas por publicación (cP), con una media de 5,42 y un valor máximo de 17,22, o bien el número máximo de citas recibidas por la publicación más citada (McP), con una media de 23,20 y un máximo de 108.

En la tabla II se presentan las correlaciones existentes entre los indicadores bibliométricos considerados.

TABLA II

Correlaciones $r$ entre los diferentes indicadores estudiados

\begin{tabular}{c|c|c|c|c|c|c|c|c|c}
\hline $\mathbf{h}$ & $\mathbf{m}$ & $\mathbf{n P}$ & $\mathbf{P a}$ & $\mathbf{c}$ & $\mathbf{c P}$ & $\mathbf{a}$ & $\mathbf{c / a}$ & $\mathbf{M c P}$ & $\mathbf{\%} \mathbf{~ N C}$ \\
\hline 1 & 0,275 & 0,854 & 0,766 & 0,881 & 0,553 & 0,556 & 0,877 & 0,797 & $-0,492$ \\
\hline & 1 & 0,090 & 0,459 & 0,199 & 0,391 & $-0,424$ & 0,482 & 0,287 & $-0,291$ \\
\hline & & 1 & 0,836 & 0,914 & 0,245 & 0,587 & 0,786 & 0,609 & $-0,276$ \\
\hline & & & 1 & 0,776 & 0,252 & 0,206 & 0,862 & 0,587 & $-0,245$ \\
\hline & & & & 1 & 0,411 & 0,448 & 0,888 & 0,726 & $-0,296$ \\
\hline & & & & & 1 & 0,082 & 0,609 & 0,764 & $-0,598$ \\
\hline & & & & & & 1 & 0,226 & 0,337 & $-0,296$ \\
\hline & & & & & & & 1 & 0,823 & $-0,400$ \\
\hline & & & & & & & & 1 & $-0,434$ \\
\hline & & & & & & & & & 1 \\
\hline
\end{tabular}


Los resultados de las correlaciones obtenidas concuerdan esencialmente con los obtenidos por otros autores (Cronin y Meho, 2006) (Saad, 2006) (van Ran, 2006) (Costas y Bordons, 2007), en el sentido que el indice h tiene una relación lineal intensa (r 0,6) con:

a) Número de publicaciones (nP).

b) Número de publicaciones por año (Pa).

c) Número de citas (c).

d) Número de citas por año $(\mathbf{c} / \mathbf{a})$.

e) Número de citas recibidas por la publicación más citada (McP).

y una correlación lineal notable $(0,4 \leq \mathrm{r}<0,6)$ con:

a) Número de citas por publicación (cP).

b) Número de años de actividad (a).

c) Porcentajes de publicaciones nunca citadas -correlación negativa- (\% NC).

La correlación entre el indice $b$ y el indice $m$ es débil, lo que significa que la intensidad de la asociación lineal es baja.

Respecto a los otros indicadores se observa una correlación lineal intensa (r 0,6) entre:

a) Número de publicaciones ( $\mathbf{n P})$ y número de publicaciones por año $(\mathbf{P a})$.

b) Número de publicaciones (nP) y número de citas recibidas (c).

c) Número de publicaciones (nP) y número de citas por año $(\mathbf{c} / \mathbf{a})$.

d) Número de publicaciones ( $\mathbf{n P}$ ) y número de citas recibidas por publicación más citada (McP).

e) Número de publicaciones por año (Pa) y número de citas recibidas (c).

f) Número de publicaciones por año $(\mathbf{P a})$ y número de citas por año $(\mathbf{c} / \mathbf{a})$.

g) Número de citas recibidas (c) y número de citas por año (c/a).

b) Número de citas recibidas (c) y número de citas recibidas por publicación más citada (McP).

i) Número de citas por publicación $(\mathbf{c P})$ y número de citas por año $(\mathbf{c} / \mathbf{a})$.

j) Número de citas por publicación $(\mathbf{c P})$ y número de citas recibidas por publicación más citada (McP).

k) Número de citas por año (c/a) y número de citas recibidas por publicación más citada (McP).

En la tabla III se presentan los valores medios de los diferentes indicadores estudiados por institución, y conviene señalar, otra vez, que cada institución representa una población - la totalidad de los investigadores - y no una muestra.

Según se muestra en la tabla III, el Consejo Superior de Investigaciones Científicas (CSIC) tiene un indice $b$ medio de 9,89, muy superior a los valores de las otras instituciones, ello se explicaría por el hecho que la dedicación a la actividad 


\section{TABLA III}

Comparación de la media aritmética de 10 indicadores bibliométricos entre las instituciones a las que pertenecen los investigadores textiles españoles estudiados

\begin{tabular}{l|c|c|c|c|c|c|c|c|c|c}
\hline Institución & $\mathbf{h}$ & $\mathbf{m}$ & $\mathbf{n P}$ & $\mathbf{P a}$ & $\mathbf{c}$ & $\mathbf{c P}$ & $\mathbf{a}$ & $\mathbf{c / a}$ & $\mathbf{M c P}$ & $\mathbf{\%} \mathbf{~ N C}$ \\
\hline UPC & 4,47 & 0,32 & 16,69 & 1 & 84,84 & 5,18 & 18,81 & 5,37 & 23,5 & 29,68 \\
\hline UPV & 3,75 & 0,57 & 10,17 & 1,39 & 72,17 & 4,29 & 6,08 & 6,79 & 12,33 & 38,87 \\
\hline US & 1,50 & 0,12 & 3,5 & 0,26 & 9,50 & 2,42 & 15,00 & 0,89 & 4,50 & 3,33 \\
\hline CSIC & 9,89 & 0,50 & 60,21 & 2,52 & 462,84 & 7,36 & 21,47 & 19,76 & 37,63 & 17,71 \\
\hline \multirow{2}{*}{ AITEX } & 1,57 & 0,71 & 2,86 & 1,13 & 15,86 & 4,54 & 2,43 & 4,64 & 11,14 & 58,98 \\
\hline \multirow{2}{*}{ AITA } & 2,50 & 0,27 & 9,5 & 0,78 & 22,00 & 1,74 & 11,00 & 1,44 & 4,00 & 33,82 \\
\hline
\end{tabular}

investigadora es exclusiva a diferencia del profesorado universitario que debe de compartir su tiempo con la actividad docente. Y también obtiene valores superiores en los otros indicadores, excepto el indicador $\mathbf{m}$ y el indicador $\mathbf{\%} \mathbf{~ N C} \mathbf{c u}-$ yos valores más altos los obtiene el Instituto Tecnológico de Alcoy (AITEX).

En la tabla IV se compara los resultados de las correlaciones del estudio (tabla II) con las obtenidas por otros autores, y se observa que, a pesar de trabajar con muestras de tamaños bastante distintos - en nuestro caso de los investigadores textiles, trabajamos con la población-, la correlación entre el indice $b$ con el número de citas es muy intensa.

\section{TABLA IV}

Comparación de las correlaciones obtenidas por varios autores entre el índice b $y$ otros indicadores

\begin{tabular}{|c|c|c|c|c|c|c|}
\hline & $\begin{array}{c}\text { Estudio } \\
\text { Textil- } \\
\text { España } \\
2011\end{array}$ & $\begin{array}{l}\text { Grupo } \\
\text { SCIMAGO } \\
2007\end{array}$ & $\begin{array}{l}\text { Salgado y } \\
\text { otros, } \\
2007\end{array}$ & $\begin{array}{l}\text { van Raan } \\
\text { A. F. } 2006\end{array}$ & $\begin{array}{c}\text { Saad, G. } \\
2006\end{array}$ & $\begin{array}{l}\text { Costas y } \\
\text { Bordons } \\
2007\end{array}$ \\
\hline$b$ con . $^{\circ}$ publicaciones & 0,854 & 0,497 & - & 0,696 & - & 0,903 \\
\hline$b \operatorname{con} n .^{\circ}$ citas & 0,881 & 0,981 & 0,880 & 0,937 & 0,870 & 0,963 \\
\hline$b$ con citas/publicaciones & 0,553 & 0,649 & - & 一 & - & - \\
\hline $\begin{array}{l}b \text { con } \mathrm{n}^{\circ} \text { citas recibidas } \\
\text { por la publicación más } \\
\text { citada }\end{array}$ & 0,790 & - & 0,610 & - & - & - \\
\hline Citas con $n .^{\circ}$ publicaciones & 0,914 & 0,368 & - & - & - & 0,888 \\
\hline Muestra (n. ${ }^{\circ}$ de autores) & $73^{*}$ & 10 & 58 & 147 & 55 & 348 \\
\hline
\end{tabular}

* Se trata de la población y no de la muestra. 
Tanto en este estudio como en el de van Raan (2006) la correlación entre el indice $b$ con el número de publicaciones es intensa, sin embargo, estos valores divergen con el estudio realizado por el Grupo SCIMAGO (2007) sobre algunos destacados científicos españoles debido, principalmente, al pequeño tamaño de su muestra (10 investigadores). En el estudio de SCIMAGO se obtuvo que la correlación entre el índice $b$ y la cantidad de publicaciones era de 0,497 $\left(R^{2}=0,248\right)$, un valor que según los autores es suficientemente bajo teniendo en cuenta el tamaño de muestra utilizado como para afirmar que no había ninguna relación entre ambos indicadores.

La elevada correlación entre el índice $b$ y el número de citas que se ha encontrado en este trabajo, también se ha observado en los estudios antes citados.

Cuando se compara el índice $b$ con la citación media (citas/publicaciones), se observa una cifra más baja, con un $0,649\left(\mathrm{R}^{2}=0,422\right)$ para el estudio del Grupo SCIMAGO y 0,553 para nuestro estudio, lo que parece indicar que en el primer caso estamos ante variables independientes y no así en este estudio que muestra una relación lineal notable.

La correlación intensa entre el indice $b$ y número de citas recibidas por la publicación más citada obtenida en el presente estudio es similar a la del Grupo SCIMAGO.

Cuando se correlacionan citas y publicaciones, el Grupo SCIMAGO (2007) obtiene una correlación de $0,368\left(\mathrm{R}^{2}=0,136\right)$ que nos indica, para este tamaño de muestra, que estamos ante variables independientes, sin embargo en este estudio se trabaja con la población y no con una muestra; la correlación es intensa 0,917 .

Finalmente, se ha procedido a realizar un análisis factorial de los indicadores del presente estudio (73 investigadores y 9 indicadores) para poner de manifiesto la estructura latente de los indicadores (todos menos el indice b); esta estructura está formada por tres factores que explican el 87,64\% de la varianza de las variables originales (indicadores). La tabla $\mathrm{V}$ muestra las cargas factoriales para cada indicador del estudio, y con un asterisco se remarca las cargas con mayor peso en cada factor (las mayores de 0,5).

El factor 1 (F1) queda claramente configurado por los indicadores $\mathrm{nP}, \mathrm{Pa}, \mathrm{c}$ y c/a, el factor 2 (F2) por cP, McP y \% NC, y el factor 3 (F3) por m, y a. El primer factor se le podría etiquetar como un balance producción-reconocimiento, el segundo como la utilidad científica relativa y el tercero tiene una dimensión temporal y podría etiquetarse por tiempo de actividad investigadora.

El análisis de regresión entre el indice $b$ estandarizado $\left(\mathrm{h}_{\text {std }}\right)$ y las puntuaciones factoriales conduce al siguiente modelo:

$$
\begin{gathered}
\mathrm{h}_{\mathrm{std}}=0,815 \text { Balance producción-reconocimiento }+0,468 \text { Utilidad científica } \\
\text { relativa }-0,162 \text { Tiempo actividad }
\end{gathered}
$$

$$
\mathrm{R}^{2}(\text { ajustado) }=90,58 \%
$$




\section{TABLA V}

Matriz de factores rotados por el procedimiento Varimax

\begin{tabular}{l|c|c|c|c}
\hline \multicolumn{1}{c|}{ Indicador } & F1 & F2 & F3 & Comunalidad \\
\hline $\mathrm{m}$ & 0,2900 & 0,2980 & $0,8271^{*}$ & 0,8570 \\
\hline $\mathrm{nP}$ & $0,9298^{*}$ & 0,1227 & $-0,2808$ & 0,9584 \\
\hline $\mathrm{Pa}$ & $0,9332^{*}$ & 0,0821 & 0,1864 & 0,9124 \\
\hline $\mathrm{c}$ & $0,9112^{*}$ & 0,2536 & $-0,1387$ & 0,9132 \\
\hline $\mathrm{cP}$ & 0,2065 & $0,8957^{*}$ & 0,1644 & 0,8721 \\
\hline $\mathrm{a}$ & 0,3530 & 0,2079 & $-0,8493^{*}$ & 0,8893 \\
\hline $\mathrm{c} / \mathrm{a}$ & $0,8682^{*}$ & 0,4330 & 0,1735 & 0,9630 \\
\hline $\mathrm{McP}$ & 0,6219 & $0,6466^{*}$ & $-0,0190$ & 0,8052 \\
\hline$\% \mathrm{NC}$ & $-0,1074$ & $0,8362^{*}$ & 0,0761 & 0,7167 \\
\hline
\end{tabular}

* Cargas con mayor peso en cada factor.

Este modelo explica el $90,58 \%$ de la variabilidad del indice $b$ con los indicadores del presente estudio. Por tanto, el indice $b$ se puede explicar por tres macroindicadores (factores latentes) independientes que ponen de manifiesto tres dimensiones fundamentales del índice.

\section{Conclusiones}

El estudio analiza, por primera vez, el indice $h$ de los investigadores textiles de un país (España) y aporta información, también por primera vez, del rango de valores de este índice en el área de conocimiento textil.

Dado que los investigadores textiles constituyen un colectivo muy heterogéneo formado por ámbitos de estudio muy distintos, la utilización del índice $b$ para evaluar la trayectoria investigadora de este colectivo puede dar lugar a interpretaciones erróneas puesto que en realidad se están analizando áreas de conocimiento muy distintas e incomparables (la investigación sobre depuración de aguas tiene poco que ver con la hilatura, la confección de prendas o la química de los tensoactivos, por poner sólo un ejemplo, a pesar que en todos estos casos haya como común denominador la industria textil). Por otra parte tal como han indicado varios autores, Hirsch (2005), Iglesias y Pecharomán (2006), Costas y Bordons (2007), Imperial y Rodríguez-Navarro (2009), etc., el índice h no parece ser el indicador más adecuado para evaluar el nivel científico, trayectoria o carrera de colectivos muy heterogéneos.

El indice $b$ presenta también limitaciones cuando se usa para comparar investigadores cuya actividad principal se ha producido en momentos históricos en los que las políticas científicas del país han sido claramente distintas. Mientras que actualmente en España la presión por publicar es muy elevada no era así 
hace tan solo 25 años atrás (Imperial y Rodríguez-Navarro, 2009). Esto explicaría que algunos investigadores españoles con una trayectoria relativamente corta presenten actualmente unos valores de indice $b$ muy superiores a colegas de mucha mayor edad. En este caso es más adecuado determinar el indice $b$ de un período de tiempo determinado como por ejemplo de los últimos 10 años, tal como propone el propio Hirscht (2005). También es cierto que una gran parte de los investigadores españoles de mayor edad, especialmente de departamentos universitarios pequeños como son los de ingeniería textil en España, han tenido que asumir una mayor cuota de dedicación a funciones de gestión, que por regla general repercute negativamente en la producción científica, y en definitiva al indice $b$.

En este estudio el índice $h$ presenta una correlación positiva, entre intensa y notable, con el número de publicaciones $(\mathrm{nP})$, publicaciones por año (Pa), número de citas recibidas (c), citas por año (c/a), número de citas recibidas por la publicación más citada (McP). La correlación es notable pero de signo negativo con el porcentaje de publicaciones de la obra del autor que no han sido citadas nunca (\% NC).

Los resultados de este estudio muestran las diferencias de indice $b$ entre los investigadores de instituciones dedicadas al 100\% a la investigación y las que comparten la investigación con otras actividades como docencia, gestión y transferencia de tecnología. Así por ejemplo, el Consejo Superior de Investigaciones Científicas (CSIC) obtiene valores superiores a las otras instituciones en todos los indicadores excepto el índice $b$ por año de actividad (m) y el porcentaje de publicaciones de la obra del autor que no han sido citadas nunca (\% NP), cuyos valores más altos se presentan en el Instituto Tecnológico Textil de Alcoy (AITEX).

El indice $b$ obtenido en este estudio puede explicarse de forma satisfactoria mediante tres factores independientes:

a) balance producción-reconocimiento (número de publicaciones, número de publicaciones por año de actividad y número de citas por año de actividad).

b) utilidad científica relativa (número de citas por publicación, porcentaje de publicaciones del autor que no han sido citadas y número de citas recibidas por la publicación más citada).

c) tiempo de actividad (índice $b$ por año de actividad y años transcurridos entre primera y última publicación).

\section{Bibliografía}

Buela-Casal, G. (2001). La Psicología española y su proyección internacional. El problema del criterio: Internacional, calidad y castellano y/o inglés. Papeles Del Psicólogo, vol. 79, 53-57. 
Buela-Casal, G. (2002). La evaluación de la Investigación Científica: El criterio de la opinión de la mayoría, El Factor de Impacto, El Factor de Prestigio y "Los Diez Mandamientos" para incrementar las citas. Análisis y Modificación de la Conducta, vol. 28, 455-476.

Buela-Casal, G. (2003). Evaluación de la calidad de los artículos y de las Revistas Científicas: Propuesta del Factor de Impacto Ponderado y de un Índice de Calidad. Psicothema, vol. 15, n. ${ }^{\circ} 1,23-35$.

Buela-Casal, G., y otros. (2006). Measuring Internationality: Reflections and Perspectives on Academic Journals. Scientometrics, 2006, vol. 67, 45-65.

Costas, R., y Bordons, M. (2007). Una visión crítica del Indice h: algunas consideraciones derivadas de su aplicación práctica. El Profesional de la Información, vol. 16, no. 5, 427-432.

Cronin, B.,y Meho, L. I. (2006). Using the h-index to rank influential information scientists. Journal of the American Society for Information Science and Technology, vol. 57, n. ${ }^{\circ}$ 9, $1275-1278$.

Garfield, E. (1955). Citation Index for Science: A New Dimension in Documentation through Association of Ideas. SCIENCE, vol. 12, 108-111.

Garfield, E. (1984). Essays of an information scientist. ISI Press ed., Philadelphia: ISI Press. How to use Citation Analysis for Faculty Evaluations, and when is it Relevant? Parts 1 and 2., 354-372.

Garfield, E. (1996). Fortnightly Review: How can Impact Factors be Improved? British Medical Journal, vol. 313, 411-413.

Grupo SCIMAGO. (2007). El Índice $b$ de Hirsch: su aplicación a algunos de los científicos españoles más destacados. El Profesional de la Información, vol. 16, n. ${ }^{\circ}$ 1, 47-49.

Hirsch, J. E. (2005). An Index to Quantify an individual's Scientific Output. Proceedings of the National Academy of the Sciences USA, vol. 102, 16.569-16.572.

Iglesias, J. E., y Pecharromán, C. (2006). Scaling the h-Index for Different Scientific Fields. http://arxiv.org/pdf/physics/0607224

Imperial, J., y Rodríguez-Navarro, A. (2009). Utilidad del Índice h de Hirsch para evaluar la investigación en España. $M i+d$. http://www.madrimasd.org/informacionIdi/analisis/ analisis/analisis. asp? $i \mathrm{~d}=24360 \& \mathrm{sec}=2$

ISI. (1999). Citations Reveal Concentrated Influences: Some Fields have it, but what does it means. Science Watch, vol. january-february, n. ${ }^{\circ} 1$.

Kelly, C. D., y Jennions, M. D. (2006). The h-Index and Career Assessment by Numbers. Trends in Ecology and Evolution, vol. 21, n. ${ }^{\circ} 4,167-170$.

Nieminen, P., y otros. (2006). The Relationship between Quality of Research and Citation Frequency. BMC Medical Research, 2006, vol. 6, 42.

Pritcchard, A. (1969). Statiscal Bibliography Or Bibliometrics? Journal of Documentation, vol. 25, n. ${ }^{\circ} 4$, 348-349.

Salgado, J. F., y Páez, D. (2007). La poductividad científica y el Índice h de Hirchs de la Psicología Social Española: Convergencia entre Indicadores de productividad y comparación con otras áreas. Psicothema, vol. 19, 179-189.

Seglen,O. P. (1992). How Representative is the Journal Impact Factor? Research Evaluation, vol. 2, 143-149. 
Seglen, O. P. (1997). Why the Impact Factor Journals should Not be used for Evaluating Research. BMJ, 1997, vol. 314, 498-502.

Seglen, O. P. (1998). Citation and Journal Impact Factors are Not Suitable for Evaluation of Research. Acta Orthophedica Scandinavia, 1998, vol. 69, 224-229.

Simkim, M. V., y Rovchowdhhury, V. P. (2003). Read before You Cite! Complex Syst, vol. 14, 269-274.

van Raan, A. F. J. (2006). Comparisons of the Hirsch-Index with Standard Bibliometric Indicators and with Peer Judgment for 147 Chemistry Research Groups. Scientometrics, vol. 67, n. $^{\circ} 3,491-502$. 